\title{
Sensor/Actuator Faults Detection for Networked Control Systems via Predictive Control
}

\author{
Yu-Yan Zhang Jun-Ling Zhang Xiao-Yuan Luo Xin-Ping Guan \\ Institute of Electrical Engineering, Yanshan University, Qinhuangdao 066004, China
}

\begin{abstract}
Quantized fault detection for sensor/actuator faults of networked control systems (NCSs) with time delays both in the sensor-to-controller channel and controller-to-actuator channel is concerned in this paper. A fault model is set up based on the possible cases of sensor/actuator faults. Then, the model predictive control is used to compensate the time delay. When the sensors and actuators are healthy, an $H_{\infty}$ stability criterion of the state predictive observer is obtained in terms of linear matrix inequality. A new threshold computational method that conforms to the actual situation is proposed. Then, the thresholds of the false alarm rate (FAR) and miss detection rate (MDR) are presented by using our proposed method, which are also compared with the ones given in the existing literatures. Finally, some numerical simulations are shown to demonstrate the effectiveness of the proposed method.
\end{abstract}

Keywords: Networked control system, fault detection, false alarm rate (FAR), miss detection rate (MDR), predictive control.

\section{Introduction}

In recent years, the issue of network-based control has drawn increasing attention of academic researchers in the area of control field. The networked control system (NCS) is defined as a feedback control system where the control loops are closed through a real-time network ${ }^{[1-3]}$, which is different from the normal control system ${ }^{[4]}$. For NCSs, there are many advantages such as low cost, reduced weight and power requirements, simple installation and maintenance, etc. Because of the introduction of communication network, there are some issues that need to bring to the attention, e.g., network-induced delay, packet loss, the effects of quantization, etc. As a result, conventional control theories must be re-evaluated before being applied to NCSs.

Since fault detection (FD) technique is essential to improve the safety and reliability of dynamic systems ${ }^{[5]}$, more and more attention has been paid to $\mathrm{FD}$ of $\mathrm{NCSs}^{[6-8]}$. Wang et al. ${ }^{[6]}$ studied the fault detection of NCSs with both access constraints and random packet dropout, where the schedule of the access to the networks was characterized by periodic communication sequence. In [7], the problem of $H_{\infty}$ fault detection filter design for a class of networked control systems was investigated through describing time delay as a Markov process. A hybrid observer-based fault detection filter (FDF) for a class of networked control systems by considering both the network-induced time delay and data packet dropout was addressed in [8]. The reviews on fault diagnosis of NCSs were summarized in [9]. Though so many works have been done on the fault detection of NCSs, Wang et al. ${ }^{[6-8]}$ considered the faults coming from the plant itself. In other word, they first gave a system suffering from sensor/actuator faults. Up to now, there is no paper that models the sensor/actuator stuck faults as a kind of fault. In this paper, a new model is proposed for

Manuscript received May 26, 2012; revised October 31, 2012

This work was supported by National Natural Science Foundation of China (No. 61074065), Natural Science Foundation of Hebei Province (No. F2012203184), and Specialized Research Fund for the Doctoral Program of Higher Education of China (No. 20111333120009). multi-input multi-output (MIMO) NCSs, where the sensor/actuator stuck faults are considered as a kind of fault. The stuck fault models can be separated by fault indicator matrices $^{[10-13]}$.

Model predictive control (MPC) can utilize the historical information to predict current and future states, so it has wide applications in dealing with the time delay and packet dropout, especially the long time delay in NCSs. In [14], an MPC strategy was proposed to overcome the data packet dropout on the sensor-to-controller channel of NCSs. Xia et al. ${ }^{[15]}$ proposed to take the latest control value from the predictive control sequence available to deal with random time delay and packet dropout, by using a networked control predictor.

In this paper, we study the FD for NCSs with disabled sensor/actuator faults and transmission time delays. For the sensor-to-controller channel time delay, a state observer is designed to compensated. And the controller-to-actuator channel time delay is compensated by using predictive controller, which is designed by optimizing one predictive performance index. Then, an $H_{\infty}$ performance analysis is established. We propose a new threshold computational method in the paper, then we give the detailed comparison with respect to the thresholds between our proposed method and the method in related literature from the point of false alarm rate/miss detection rate (FAR/MDR). A simulation example is provided to show the effectiveness of the proposed method by comparing it with the existing researches.

\section{Problem formulation}

Consider a class of MIMO discrete systems described by the following state space form

$$
\left\{\begin{array}{l}
x(k+1)=A x(k)+B u(k)+B_{1} w(k) \\
y(k)=C x(k)
\end{array}\right.
$$

where $x(k) \in \mathbf{R}^{n}$ and $u(k) \in \mathbf{R}^{m}$ are state and control inputs, $y(k) \in \mathbf{R}^{l}$ and $w(k) \in \mathbf{R}^{w}$ are system output and 
the disturbance, respectively. $A, B, B_{1}$ and $C$ are known matrices with appropriate dimensions, and $(A, C)$ is detectable.

In this paper, we consider fault detection for networked control systems via predictive control. The system diagram is shown as Fig. 1, in which the state observer and predictive controller are used.

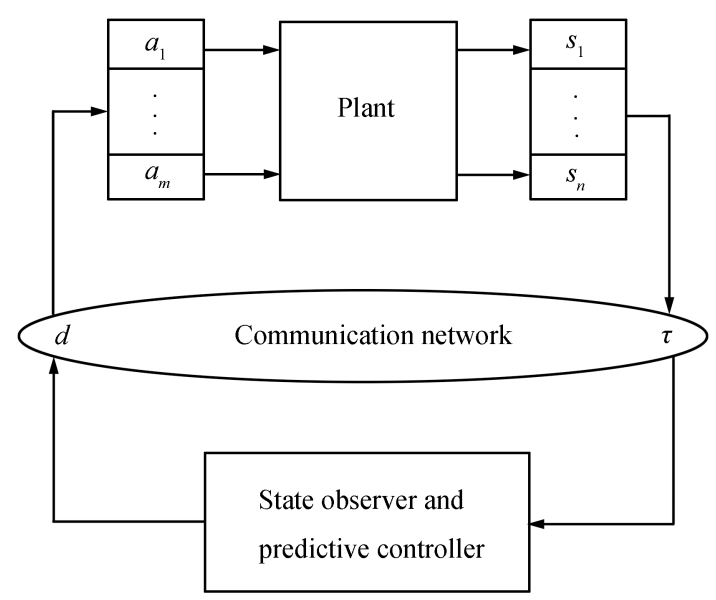

Fig. 1 The diagram of NCSs via predictive control

When all the sensors are healthy, the plant output can be transmitted successfully through the network. If some of the sensors are failed, there must exist loss of data packets.

Considering the sensor faults, a fault indicator matrix $F_{s}$ is introduced, which is given by

$$
F_{s}=\operatorname{diag}\left\{f_{s 1}, f_{s 2}, \cdots, f_{s n}\right\}
$$

with $f_{s i}=\left\{\begin{array}{l}1, \text { health } \\ 0, \text { failure }\end{array}, i=1,2, \cdots, n\right.$, indicating whether sensor $i$ is failed or not. Then, considering the various possibilities of sensor failures, the system output is

$$
\tilde{y}=F_{s r}(y(k))=F_{s r}(C x(k))
$$

where $\tilde{y} \in \mathbf{R}^{l}$ is the sensor measurement output, $F_{s r} \in$ $F_{\Theta}=\left\{F_{s 1}, F_{s 2}, \cdots, F_{s N}\right\}, r \in\{1,2, \cdots, N\}, F_{\Theta}$ is the set of sensor failure modes, with $N=2^{n}-1$, and $N$ is the number of sensor failure modes. The fault indicator matrix $F_{s r}$ is one of the failure modes, which indicates a fault mode of the sensors including only one sensor fault.

Similarly, we consider the actuator faults and let $F_{a}$ be the actuator fault indicator matrix. Here, the same as sensor faults, $F_{a}$ is given by

$$
F_{a}=\operatorname{diag}\left\{f_{a 1}, f_{a 2}, \cdots, f_{a m}\right\}
$$

with $f_{a j}=\left\{\begin{array}{ll}1, & \text { health } \\ 0, & \text { failure }\end{array} j=1,2, \cdots, m\right.$, indicating whether actuator $j$ is failed or not. Then, considering the various possibilities of actuator failures, the actuator output is

$$
\tilde{u}(k)=F_{a l}(u(k))
$$

where $\tilde{u}(k) \in \mathbf{R}^{m}$ is the actuator output, i.e., system input, $F_{a l} \in F_{\Phi}=\left\{F_{a 1}, F_{a 2}, \cdots, F_{a M}\right\}$ and $l \in\{1,2, \cdots, M\}$, $F_{\Phi}$ is the set of failure modes, with $M=2^{m}-1, M$ is the number of actuator failure modes. The fault indicator matrix $F_{a l}$ is one of the failure modes, which indicates some fault of the actuators including only one actuator fault.

Then, the model can be rewritten as

$$
\left\{\begin{array}{l}
x(k+1)=A x(k)+B u(k)+B_{f} f(k)+B_{1} w(k) \\
\tilde{y}(k)=C x(k)+D_{f} f(k)
\end{array}\right.
$$

where $B_{f}=\left[\begin{array}{ll}B F_{a l}-B & 0\end{array}\right], \quad D_{f}=\left[\begin{array}{ll}0 & F_{s r} C-C\end{array}\right]$, and $f(k)=\left[\begin{array}{ll}u(k) & x(k)\end{array}\right]^{\mathrm{T}}$.

When the packets are transmitted through the network, time delay is unavoidable. The methodology of compensating the time delay for the NCS will be proposed in the next section. Considering the networked control system, the following reasonable assumptions need to be made.

1) The plant output nodes (sensors) are clock-driven. The controller and actuators are event-driven. The data is transmitted through a single-packet.

2) The network transmission is fixed. $\tau$ and $d$ are the time delay in the sensor-to-controller channel and the controllerto-actuator channel, respectively. They are integral multiples of the sampling period.

3) The data packets can be transmitted successfully in the network.

The main objective of this paper is fault detection of sensor/actuator for NCSs with transmission time delays in the network. Firstly, we shall compensate the time delays. Then, we conduct research on fault detection by calculating the residual. Also, false alarm rate and miss detection rate, which are two propositions about fault detection, are presented.

\section{State observer and predictive con- troller design}

Since there exist time delays during the network transmission of data packets. In this section, we shall compensate these time delays by designing the state observer and predictive controller. The state observer is used to compensate the time delay in the sensor-to-controller channel by using the measured state which is transmitted through the network channel. Then based on the observer, the predictive controller is used to compensate the time delay in the controller-to-actuator channel.

In order to compensate the network transmission delay $\tau$ in the sensor-to-controller channel, $\tilde{x}(k+1 / k)$ is constructed. In the control centre, $\tilde{x}(k-\tau+1 / k-\tau)$ is received, then based on $\tilde{y}(k-\tau)$ and the input data $u(k-\tau)$ up to $u(k-1)$, we can predict the state $\tilde{x}(k / k-\tau)$.

The state observer is designed as

$$
\begin{aligned}
\tilde{x}(k+1 / k)= & A \tilde{x}(k / k-1)+B u(k)+ \\
& L(\tilde{y}(k)-C \tilde{x}(k / k-1))
\end{aligned}
$$

where $\tilde{x}(k / k-1) \in \mathbf{R}^{n}$ and $u(k) \in \mathbf{R}^{m}$ are the one-stepahead predicted state and the input of the observer at time $k$, respectively. The matrix $L \in \mathbf{R}^{n \times l}$ can be designed by observer design approach.

Based on $\tilde{y}(k-\tau)$ and the input data $u(k-\tau)$ up to $u(k-1)$ received on the side of the observer, the predictions 
of the states from time $k-\tau+1$ to $k$ are constructed as

$$
\begin{gathered}
\tilde{x}(k-\tau+1 / k-\tau)=A \tilde{x}(k-\tau / k-\tau-1)+B u(k-\tau)+ \\
L(\tilde{y}(k-\tau)-C \tilde{x}(k-\tau / k-\tau-1)) \\
\tilde{x}(k-\tau+2 / k-\tau)=A \tilde{x}(k-\tau+1 / k-\tau)+ \\
B u(k-\tau+1) \\
\cdots \\
\tilde{x}(k / k-\tau)=A \tilde{x}(k-1 / k-\tau)+B u(k-1) .
\end{gathered}
$$

Then, we can get

$$
\begin{gathered}
\tilde{x}(k / k-\tau)=A^{\tau-1}(A-L C) \tilde{x}(k-\tau / k-\tau-1)+ \\
\sum_{j=1}^{\tau} A^{\tau-j} B u(k-\tau+j-1)+A^{\tau-1} L \tilde{y}(t-\tau) .
\end{gathered}
$$

On the other hand, in order to compensate the time delay in the controller-to-actuator channel, we consider the following predictive performance index which is based on the initial state $\hat{x}(k / k-\tau)$ as

$$
\begin{aligned}
J(k)= & \sum_{i=1}^{N_{p}} \tilde{x}^{\mathrm{T}}(k+i / k-\tau) Q \tilde{x}(k+i / k-\tau)= \\
& \sum_{i=0}^{N_{u}-1} \tilde{u}^{\mathrm{T}}(k+i / k-\tau) R u(k+i / k-\tau)
\end{aligned}
$$

where $N_{p}$ is the predictive horizon, $N_{u}$ is the control horizon, $Q$ and $R$ denote positive definite weighting matrices, $\tilde{x}(k+i / k-\tau)$ is the predicted state at time $k+i$, and $u(k+i / k-\tau)$ is the corresponding predicted control input at time $k+i$. It is assumed that the control increments are zero beyond the control horizon, i.e.,

$$
u(k+i / k-\tau)=u\left(k+N_{u}-1 / k-\tau\right), i \geqslant N_{u}-1 .
$$

Let

$$
\begin{aligned}
& \tilde{X}(k+1)=\left[\begin{array}{lll}
\tilde{x}(k+1 / k-\tau) & \cdots & \tilde{x}\left(k+N_{p} / k-\tau\right)
\end{array}\right]^{\mathrm{T}} \\
& U(k)=\left[\begin{array}{lll}
u^{\mathrm{T}}(k / k-\tau) & \cdots & u^{\mathrm{T}}\left(k+N_{u}-1 / k-\tau\right)
\end{array}\right]^{\mathrm{T}} \\
& A_{p}=\left[\begin{array}{llll}
A^{\mathrm{T}} & \left(A^{2}\right)^{\mathrm{T}} & \cdots & \left(A^{N_{p}}\right)^{\mathrm{T}}
\end{array}\right]^{\mathrm{T}} \\
& B_{p}= \\
& {\left[\begin{array}{cccc}
B & 0 & \cdots & 0 \\
A B & B & \cdots & 0 \\
\cdots & & \cdots & \cdots \\
A^{N_{p}-1} B & A^{N_{p}-2} B & \cdots & A^{N_{p}-N_{u}} B+A^{N_{p}-N_{u}-1} B+\cdots+B
\end{array}\right] .}
\end{aligned}
$$

We have

$$
\tilde{X}(k+1)=A_{p} \tilde{x}(k / k-\tau)+B_{p} U(k) .
$$

Substituting (9) into (7), we have

$$
\begin{aligned}
J(k)= & \left(A_{p} \tilde{x}(k / k-\tau)+B_{p} U(k)\right)^{\mathrm{T}} Q \times \\
& \left(A_{p} \tilde{x}(k / k-\tau)+B_{p} U(k)\right)+U(k)^{\mathrm{T}} R U(k)
\end{aligned}
$$

where $Q=\operatorname{diag}\{Q, \cdots, Q\}, R=\operatorname{diag}\{R, \cdots, R\}$

Then by minimizing (10), we can obtain the optimal predictive control sequence as

$$
\begin{aligned}
U^{*}(k)= & -\left(B_{p}^{\mathrm{T}} Q B_{p}+R\right)^{-1} B_{p}^{\mathrm{T}} Q A_{p} \tilde{x}(k / k-\tau) \triangleq \\
& F \tilde{x}(k / k-\tau) .
\end{aligned}
$$

If the time delay in the controller-to-actuator channel is $d$, the actuator can be chosen as

$$
u(k+d / k-\tau)=E F \tilde{x}(k / k-\tau)
$$

where $E=\left[\begin{array}{lll}0_{m \times(d-2) m} & I_{m \times m} & 0_{m \times\left(N_{u}-d+1\right) m}\end{array}\right]$.

For notational simplicity, denote $K=E F$, we get

$$
u(k+d / k-\tau)=K \tilde{x}(k / k-\tau) .
$$

Since there exist the sensor-to-controller channel delay $\tau$ and the controller-to-actuator channel delayd, in order to analyze conveniently, let $\tilde{x}(k-d / k-\tau-d)$ represent $\tilde{x}(k / k-\tau)$, the control input and plant state vectors are given by the following equality.

$$
\begin{aligned}
& u(k)=u(k / k-\tau-d)=K \tilde{x}(k-d / k-\tau-d) \\
& x(k+1)=A x(k)+ \\
& B K\left(A^{\tau-1}(A-L C) \tilde{x}(k-\tau / k-\tau-1)+\right. \\
& \left.\sum_{j=1}^{\tau} A^{\tau-j} B u(k-\tau+j-1)+A^{\tau-1} L \tilde{y}(t-\tau)\right)+ \\
& B_{1} w(k) .
\end{aligned}
$$

When considering the FD of NCS with network-induced delay, the FD filter can be constructed as

$$
\left\{\begin{array}{l}
\hat{x}(k+1)=A \hat{x}(k)+ \\
B K\left(A^{\tau-1}(A-L C) \tilde{x}(k-\tau / k-\tau-1)+\right. \\
\sum_{j=1}^{\tau} A^{\tau-j} B u(k-\tau+j-1)+A^{\tau-1} L \tilde{y} \times \\
(t-\tau))+L(\tilde{y}(k)-C \tilde{x}(k / k-1)) \\
r_{k}=V(y(k)-\hat{y}(k)) \\
\hat{y}(k)=C \hat{x}(k)
\end{array}\right.
$$

where $r_{k}$ is the residual error vector, and $V$ is the residual error output matrix.

Let $e(k)=x(k)-\hat{x}(k / k-1)$, when all the sensors and actuators are healthy, we can get the state error dynamics through comparing (14) with (15) as

$$
e(k+1)=(A-L C) e(k)+B_{1} w(k) .
$$

Combining (14)-(16), we have

$$
\left\{\begin{array}{l}
Z(k+1)=\Omega Z(k)+B_{w} w(k) \\
r(k)=\tilde{V} C Z(k)
\end{array}\right.
$$

where

$$
\begin{aligned}
& Z(k)=\left[\begin{array}{llll}
x(k) & \cdots & x(k-\tau-d) & \cdots
\end{array}\right. \\
& u(k-1) \quad \cdots \quad u(k-\tau-d) \quad \tilde{x}(k / k-1) \cdots \\
& \tilde{x}(k-\tau-d / k-\tau-d-1) \quad e(k)]
\end{aligned}
$$


$\Omega=\left[\begin{array}{cccc}\Omega_{11}(\tau, d) & \Omega_{12}(\tau, d) & \Omega_{13}(\tau, d) & \Omega_{14}(\tau, d) \\ \Omega_{21}(\tau, d) & \Omega_{22}(\tau, d) & \Omega_{23}(\tau, d) & \Omega_{24}(\tau, d) \\ \Omega_{31}(\tau, d) & \Omega_{32}(\tau, d) & \Omega_{33}(\tau, d) & \Omega_{34}(\tau, d) \\ \Omega_{41}(\tau, d) & \Omega_{42}(\tau, d) & \Omega_{43}(\tau, d) & \Omega_{44}(\tau, d)\end{array}\right]$

$\Omega_{11}(\tau, d)=\left[\begin{array}{ccccc}A & 0 & \cdots & 0 & \Lambda_{1} \\ I & 0 & \cdots & 0 & 0 \\ & \cdots & \cdots & \multicolumn{2}{c}{\cdots} \\ 0 & 0 & \cdots & 0 & 0 \\ 0 & 0 & \cdots & I & 0\end{array}\right]$

$\Omega_{12}(\tau, d)=\left[\begin{array}{ccccc}\Lambda_{2} & \Lambda_{3} & \cdots & \Lambda_{4} & \Lambda_{5} \\ 0 & 0 & \cdots & 0 & 0 \\ & \cdots & \cdots & \multicolumn{2}{c}{\cdots} \\ 0 & 0 & \cdots & 0 & 0 \\ 0 & 0 & \cdots & 0 & 0\end{array}\right]$

$\Omega_{13}(\tau, d)=\left[\begin{array}{ccccc}0 & 0 & \cdots & 0 & \Lambda_{6} \\ 0 & 0 & \cdots & 0 & 0 \\ \cdots & \cdots & \multicolumn{2}{c}{\cdots} \\ 0 & 0 & \cdots & 0 & 0 \\ 0 & 0 & \cdots & 0 & 0\end{array}\right]$

$\Omega_{21}(\tau, d)=\left[\begin{array}{ccccc}0 & 0 & \cdots & 0 & \Lambda_{7} \\ 0 & 0 & \cdots & 0 & 0 \\ \cdots & \cdots & \multicolumn{2}{c}{\cdots} \\ 0 & 0 & \cdots & 0 & 0 \\ 0 & 0 & \cdots & 0 & 0\end{array}\right]$

$\Omega_{22}(\tau, d)=\left[\begin{array}{ccccc}\Lambda_{8} & \Lambda_{9} & \cdots & \Lambda_{10} & \Lambda_{11} \\ I & 0 & \cdots & 0 & 0 \\ & \cdots & \cdots & & \cdots \\ 0 & 0 & \cdots & 0 & 0 \\ 0 & 0 & \cdots & I & 0\end{array}\right]$

$\Omega_{23}(\tau, d)=\left[\begin{array}{ccccc}0 & 0 & \cdots & 0 & \Lambda_{12} \\ 0 & 0 & \cdots & 0 & 0 \\ \cdots & \cdots & \cdots & \cdots \\ 0 & 0 & \cdots & 0 & 0 \\ 0 & 0 & \cdots & 0 & 0\end{array}\right]$

$\Omega_{31}(\tau, d)=\left[\begin{array}{ccccc}\Lambda_{13} & 0 & \cdots & 0 & \Lambda_{14} \\ 0 & 0 & \cdots & 0 & 0 \\ & \cdots & \cdots & \multicolumn{2}{c}{\cdots} \\ 0 & 0 & \cdots & 0 & 0 \\ 0 & 0 & \cdots & 0 & 0\end{array}\right]$

$\Omega_{32}(\tau, d)=\left[\begin{array}{ccccc}\Lambda_{15} & \Lambda_{16} & \cdots & \Lambda_{17} & \Lambda_{18} \\ 0 & 0 & \cdots & 0 & 0 \\ & \cdots & \cdots & & \cdots \\ 0 & 0 & \cdots & 0 & 0 \\ 0 & 0 & \cdots & 0 & 0\end{array}\right]$

$\Omega_{33}(\tau, d)=\left[\begin{array}{ccccc}\Lambda_{19} & 0 & \cdots & 0 & \Lambda_{20} \\ I & 0 & \cdots & 0 & 0 \\ & \cdots & \cdots & \multicolumn{2}{c}{\cdots} \\ 0 & 0 & \cdots & 0 & 0 \\ 0 & 0 & \cdots & I & 0\end{array}\right]$
$\Omega \in \mathbf{R}^{(2(\tau+d+1) n+(\tau+d) m) \times(2(\tau+d+1) n+(\tau+d) m)}$

$\Omega_{11}(\tau, d) \in \mathbf{R}^{(\tau+d+1) n \times(\tau+d+1) n}$

$\Omega_{12}(\tau, d) \in \mathbf{R}^{(\tau+d+1) n \times(\tau+d) m}$

$\Omega_{13}(\tau, d) \in \mathbf{R}^{(\tau+d+1) n \times(\tau+d+1) n}$

$\Omega_{21}(\tau, d) \in \mathbf{R}^{(\tau+d) m \times(\tau+d+1) n}$

$\Omega_{22}(\tau, d) \in \mathbf{R}^{(\tau+d) m \times(\tau+d) m}$

$\Omega_{23}(\tau, d) \in \mathbf{R}^{(\tau+d) m \times(\tau+d+1) n}$

$\Omega_{31}(\tau, d) \in \mathbf{R}^{(\tau+d+1) n \times(\tau+d+1) n}$

$\Omega_{32}(\tau, d) \in \mathbf{R}^{(\tau+d+1) n \times(\tau+d) m}$

$\Omega_{33}(\tau, d) \in \mathbf{R}^{(\tau+d+1) n \times(\tau+d+1) n}$

$\Omega_{14}(\tau, d)=\left[\begin{array}{lllll}0 & 0 & \cdots & 0 & 0\end{array}\right]^{\mathrm{T}} \in \mathbf{R}^{(\tau+d+1) n \times n}$

$\Omega_{24}(\tau, d)=\left[\begin{array}{lllll}0 & 0 & \cdots & 0 & 0\end{array}\right]^{\mathrm{T}} \in \mathbf{R}^{(\tau+d) m \times n}$

$\Omega_{34}(\tau, d)=\left[\begin{array}{lllll}0 & 0 & \cdots & 0 & 0\end{array}\right]^{\mathrm{T}} \in \mathbf{R}^{(\tau+d+1) n \times n}$

$\Omega_{41}(\tau, d)=\left[\begin{array}{lllll}0 & 0 & \cdots & 0 & 0\end{array}\right] \in \mathbf{R}^{n \times(\tau+d+1) n}$

$\Omega_{42}(\tau, d)=\left[\begin{array}{lllll}0 & 0 & \cdots & 0 & 0\end{array}\right] \in \mathbf{R}^{n \times(\tau+d) m}$

$\Omega_{43}(\tau, d)=\left[\begin{array}{lllll}0 & 0 & \cdots & 0 & 0\end{array}\right] \in \mathbf{R}^{n \times(\tau+d+1) n}$

$\Omega_{44}(\tau, d)=A-L C$

$\Lambda_{1}=B K A^{\tau-1} L C, \quad \Lambda_{2}=B K B$

$\Lambda_{3}=B K A B, \quad \Lambda_{4}=B K A^{\tau-2} B$

$\Lambda_{5}=B K A^{\tau-1} B, \quad \Lambda_{6}=B K A^{\tau-1}(A-L C)$

$\Lambda_{7}=K A^{\tau-1} L C, \quad \Lambda_{8}=K B$

$\Lambda_{9}=K A B, \quad \Lambda_{10}=K A^{\tau-2} B$

$\Lambda_{11}=K A^{\tau-1} B, \quad \Lambda_{12}=K A^{\tau-1}(A-L C)$

$\Lambda_{13}=L C, \quad \Lambda_{14}=B K A^{\tau-1} L C$

$\Lambda_{15}=B K B, \quad \Lambda_{16}=B K A B$

$\Lambda_{17}=B K A^{\tau-2} B, \quad \Lambda_{18}=B K A^{\tau-1} B$

$\Lambda_{19}=(A-L C), \quad \Lambda_{20}=B K A^{\tau-1}(A-L C)$

$B_{w}=\left[\begin{array}{lllll}B_{1}^{\mathrm{T}} & 0 & \cdots & 0 & B_{1}^{\mathrm{T}}\end{array}\right]^{\mathrm{T}} \in \mathbf{R}^{(2(\tau+d+1) n+(\tau+d) m) \times n}$

$\tilde{V}=\left[\begin{array}{llll}0 & \cdots & 0 & V\end{array}\right]$.

\section{Main results}

\section{1 $\quad H_{\infty}$ performance analysis}

The following theorem provides a stability condition for system (17).

Theorem 1. Consider system (17), when all the sensors and actuators are healthy, the closed-loop system is stable and the $H_{\infty}$ performance with index $\gamma$ is satisfied, if there 
exists a positive definite matrix $X$ satisfying

$$
\left[\begin{array}{cccc}
-X & * & * & * \\
\tilde{V} C X & -I & * & * \\
0 & 0 & -\gamma^{2} I & * \\
\Omega X & B_{w} & 0 & -X
\end{array}\right]<0 .
$$

Proof. Let $V(k)=Z^{\mathrm{T}}(k) P Z(k)$. Then if $w(k)=0$, we have

$$
\begin{aligned}
& \Delta V(k)=V(k+1)-V(k)= \\
& \quad Z^{\mathrm{T}}(k+1) P Z(k+1)-Z^{\mathrm{T}}(k) P Z(k)= \\
& Z^{\mathrm{T}}(k)\left(\Omega^{T} P \Omega-P\right) Z(k) .
\end{aligned}
$$

If inequality (18) is satisfied, by the Schur's complement formula, we have $\Delta V(k)<0$. Therefore, system (17) is stable for $w(k)=0$.

Now, let's consider the $H_{\infty}$ performance of the closedloop system. We define the performance function as

$$
\begin{aligned}
\Upsilon \triangleq & \sum_{k=0}^{\infty}\left[r^{\mathrm{T}}(k) r(k)-\gamma^{2} w^{\mathrm{T}}(k) w(k)\right]= \\
& \sum_{k=0}^{\infty}\left[r^{\mathrm{T}}(k) r(k)-\gamma^{2} w^{\mathrm{T}}(k) w(k)+\Delta V(k)\right]+ \\
& V(0)-V(\infty) .
\end{aligned}
$$

Under the zero initial condition, i.e., $\left.V(k)\right|_{k=0}=0$ and $V(\infty) \geqslant 0$, we have

$$
\Upsilon \leqslant \sum_{k=0}^{\infty}\left[r^{\mathrm{T}}(k) r(k)-\gamma^{2} w^{\mathrm{T}}(k) w(k)+\Delta V(k)\right]
$$

in which

$$
\begin{aligned}
& r^{\mathrm{T}}(k) r(k)-\gamma^{2} w^{\mathrm{T}}(k) w(k)+\Delta V(k)=\left[\begin{array}{r}
Z(k) \\
w(k)
\end{array}\right]^{\mathrm{T}} \\
& {\left[\begin{array}{cc}
\Omega^{\mathrm{T}} P \Omega+(\tilde{V} C)^{\mathrm{T}} \tilde{V} C-P & * \\
B_{w}^{\mathrm{T}} P \Omega & B_{w}^{\mathrm{T}} P B_{w}-\gamma^{2} I
\end{array}\right]\left[\begin{array}{l}
Z(k) \\
w(k)
\end{array}\right] .}
\end{aligned}
$$

Then, $\Upsilon<0$ can be replaced by the following inequality

$$
\left[\begin{array}{cc}
\Omega^{\mathrm{T}} P \Omega+(\tilde{V} C)^{\mathrm{T}} \tilde{V} C-P & * \\
B_{w}^{\mathrm{T}} P \Omega & B_{w}^{\mathrm{T}} P B_{w}-\gamma^{2} I
\end{array}\right]<0 .
$$

By using Schur's complement formula, the following inequality can be obtained from (23).

$$
\left[\begin{array}{cccc}
-P & * & * & * \\
\tilde{V} C & -I & * & * \\
0 & 0 & -\gamma^{2} I & * \\
\Omega & B_{w} & 0 & -P^{-1}
\end{array}\right]<0 .
$$

In order to get $P$, one must linearize $(24)$ since it is a nonlinear matrix inequality. Let $X=P^{-1}$, and pre-multiplying and post-multiplying both sides of (24) by diag $\{X, I, I, I\}$, then using Schur's complement we obtain (18).

Remark 1. Theorem 1 shows that the closed-loop system (17) is stable if (18) is satisfied with $H_{\infty}$ norm bound $\gamma$. Moreover, we can obtain the disturbance rejection level $\gamma$ by solving the following optimal problem

$$
\min \alpha
$$

where $\alpha=\gamma^{2}$. It is well known that (25) is a convex optimal problem for the linear objective function, and it can be solved easily.

\subsection{Residual evaluation and threshold}

The important task for FD is the evaluation of the generated residual. One of the widely adopted approaches is to choose the so-called threshold $J_{t h}>0$ and use some properly logical relationship for fault detection.

The following 2-norm of residual signal is chosen as residual evaluation function as ${ }^{[7,16]}$

$$
J(r, k)=\left\{\sum_{k=0}^{k=L_{1}} r^{\mathrm{T}}(k) r(k)\right\}^{\frac{1}{2}}
$$

where $L_{1}$ denotes the current time step of the evaluation function.

The following threshold exists in most of the literatures $^{[7,16]}$.

$$
J_{t h}=\sup _{\bar{w}(k) \in l_{2}, B_{f}=0, D_{f}=0} \mathrm{E}\left\{\sum_{k=0}^{k=L_{t h}} r^{\mathrm{T}}(k) r(k)\right\}^{\frac{1}{2}}
$$

where $L_{t h}$ denotes the maximum time step of the evaluation function.

In this paper, the threshold is different from (28) as in $[7,16]$, and we set it as follows.

$$
\begin{array}{r}
J_{t h}(r, k)=\sup _{\bar{w}(k) \in l_{2}, B_{f}=0, D_{f}=0} \mathrm{E}(J(r, k))+ \\
\eta_{\bar{w}(k) \in l_{2}, B_{f}=0, D_{f}=0} \sigma(J(r, k))
\end{array}
$$

where $\sigma(\cdot)$ denotes the standard deviation and $\eta$ is a positive constant value related to the threshold.

$$
\begin{gathered}
\mathrm{E}(J(r, k))=\frac{1}{G} \sum_{i=1}^{n} J_{i}(r, k) \\
\sigma(J(r, k))=\sqrt{\frac{1}{G-1} \sum_{i=1}^{n}\left\{J_{i}(r, k)-\mathrm{E}(J(r, k))\right\}^{2}}
\end{gathered}
$$

where $G$ is the total test number.

Because the values of the residual evaluation function are different at different time, we would better to construct a threshold to adapt the residual evaluation function. For the random character of the disturbance, the residual signal changes in a certain range at the same time, we can perform a large number of experiments to get the proper threshold.

Remark 2. Our method is similar to the one considered in [6]. They considered the disturbance as a constant in each test, so the standard deviation of disturbance $\sigma(J(d, k))=0$. Disturbance is stochastic in practice, therefore, from a large number of experiments for seeking its mean value is more realistic.

According to the aforementioned analysis, we can draw a conclusion that the residual evaluation function may be big enough if the actuator and/or sensor are/is failed. Therefore, we can carry out fault detection via comparing the 
residual evaluation function with the threshold. The following is the criterion of fault detection.

$$
\left\{\begin{array}{l}
J(r, k)>J_{t h}(r, k), \text { if actuator and/or sensor is/are failed } \\
J(r, k) \leqslant J_{t h}(r, k), \text { if actuators and sensors are healthy. }
\end{array}\right.
$$

\subsection{FAR and MDR computation}

It is well known that the selection of the threshold is a tradeoff between FAR and $\mathrm{MDR}^{[6]}$. In order to compare the proposed threshold with the existent method, we firstly give the computing methods of FAR and MDR, and then the detailed comparison will be shown in this section.

Lemma $1^{[6]}$. Given a random variable $s$ with mean value $\bar{s}$ and variance $\sigma^{2}$, for any $\varepsilon>0$, it follows that

$$
P\{|s-\bar{s}| \geqslant \varepsilon\} \leqslant \frac{\sigma^{2}}{\varepsilon^{2}}
$$

According to (29), we say a false alarm occurs if and only if $J(r, k) \geqslant J_{t h}(r, k)$ in the absence of fault. The FAR of the threshold is determined by

$$
\begin{gathered}
P\left\{J(r, k) \geqslant J_{t h}(r, k) \mid B_{f}=0, D_{f}=0\right\}= \\
P\left\{\left\{\sum_{k=0}^{k=L_{1}} r^{\mathrm{T}}(k) r(k)\right\}^{\frac{1}{2}} \geqslant\right. \\
\bar{w}(k) \in l_{2}, B_{f}=0, D_{f}=0 \\
\quad \mathrm{E}(J(r, k))+ \\
P\left\{\left\{\sup _{\bar{w}(k) \in l_{2}, B_{f}=0, D_{f}=0} \sigma(J(r, k))\right\} \leqslant\right. \\
\left.\left.\sum_{k=0}^{k=L_{1}} r^{\mathrm{T}}(k) r(k)\right\}^{\frac{1}{2}} \geqslant\{\mathrm{E}(J(r, k))+\eta \sigma(J(r, k))\}\right\}
\end{gathered}
$$

By using Lemma 1 and (31), the FAR of threshold (28) is upper-bounded by $\frac{1}{\eta^{2}}$.

The FAR of the threshold in $[7,16]$ can be determined by

$$
\begin{aligned}
& P\left\{\left\{\sum_{k=0}^{k=L_{1}} r^{\mathrm{T}}(k) r(k)\right\}^{\frac{1}{2}} \geqslant\right. \\
& \left.\left.\left.w(k) \in \sup _{2}, B_{f}=0, D_{f}=0\right\} \sum_{k=0}^{K=L_{t h}} r^{\mathrm{T}}(k) r(k)\right\}^{\frac{1}{2}}\right\} \leqslant \\
& P\left\{\left\{\sum_{k=0}^{K=L_{1}} r^{\mathrm{T}}(k) r(k)\right\}^{\frac{1}{2}} \geqslant\right. \\
& \mathrm{E}\left\{\sum_{k=0}^{K=L_{t h}} r^{\mathrm{T}}(k) r(k)\right\}^{\frac{1}{2}}+ \\
& \\
& \left.\eta\left\{\sum_{k=0}^{K=L_{t h}} r^{\mathrm{T}}(k) r(k)\right\}^{\frac{1}{2}}\right\}
\end{aligned}
$$

where

$$
\begin{aligned}
& \mathrm{E}\left\{\sum_{k=0}^{K=L_{t h}} r^{\mathrm{T}}(k) r(k)\right\}^{\frac{1}{2}}+ \\
& \left.\eta\left\{\sum_{k=0}^{K=L_{t h}} r^{\mathrm{T}}(k) r(k)\right\}^{\frac{1}{2}}\right\} \leqslant \\
& w(k) \in \sup _{2}, B_{f}=0, D_{f}=0 \\
& \left.\sum_{k=0}^{K=L_{t h}} r^{\mathrm{T}}(k) r(k)\right\}^{\frac{1}{2}} .
\end{aligned}
$$

Here, we let $\eta=\left(J_{t h}-E(J(r, k))\right) / \sigma(J(r, k))$.

By using Lemma 1 , the FAR of the threshold in $[7,16]$ is upper-bounded by $\frac{1}{\eta^{2}}$.

Ideal fault detection method for a system is that the evaluation function should have been greater than the threshold when a fault occurred. We introduce the MDR computation given by [17] as

$$
M D R=\frac{G_{1}}{G \times G_{2}}
$$

where $G$ is the total test number, $G_{1}$ is the total time of the faulty system during which the value of evaluation function remains less than the threshold in all simulations, and $G_{2}$ is the total fault time in each test.

\section{Simulation study}

In this section, a simple linear time-invariant system is constructed to illustrate our method, and we shall show the detailed comparison of FAR/MDR in this section.

Consider system (1) with

$$
\begin{aligned}
& A=\left[\begin{array}{ll}
-0.01 & 0.1 \\
-0.3 & 0.1
\end{array}\right], B=\left[\begin{array}{ll}
0.1 & 0.2 \\
0.3 & 0.5
\end{array}\right], B_{1}=\left[\begin{array}{l}
0.2 \\
0.1
\end{array}\right] \\
& C=\left[\begin{array}{cc}
0.1 & 0 \\
0 & 0.3
\end{array}\right], V=\left[\begin{array}{ll}
0.5 & 0.5
\end{array}\right] .
\end{aligned}
$$

Matrix $L$ is designed by pole assignment to ensure the closed-loop system without network delay is stable. With the poles placed as $\left[\begin{array}{ll}0.5 & 0.01\end{array}\right]$, we design $L$ as

$$
L=\left[\begin{array}{cc}
-5.1000 & 0.333 \\
-3.000 & 0.3000
\end{array}\right] \text {. }
$$

Given the desired parameter matrices as

$$
Q=\left[\begin{array}{ll}
2 & 0 \\
0 & 2
\end{array}\right], \quad R=\left[\begin{array}{ll}
1 & 0 \\
0 & 1
\end{array}\right]
$$

when the time delay $d=1$, matrix $K$ is designed as

$$
K=\left[\begin{array}{ll}
0.0663 & -0.0287 \\
0.1097 & -0.0509
\end{array}\right] \text {. }
$$

For $\tau=2$, solving the optimal problem (25), we can get the disturbance rejection level $\gamma=0.036$.

In this simulation, we carry out 200 times of simulation experiments. And in each experiment, we set the time of fault occurrence is $15 \mathrm{~s}$, Hence, $G=200, G_{2}=15$. In addition, we set the allowable range of FAR as $6.25 \%$, i.e., $\eta=4$. Then, we can compute $\mathrm{E}(J(r, k)), \sigma(J(r, k)), J_{t h}(r, k)$ by using (28) and (29) at $20 \mathrm{~s}, 60 \mathrm{~s}, 100 \mathrm{~s}$ and $140 \mathrm{~s}$, respectively. Using $\frac{1}{\eta^{2}}$ and (33), we get the values of FAR and MDR. The values of FAR and MDR in $[7,16]$ are also computed. They are all listed in Table 1.

From Table 1, it can be concluded that the FARs of our proposed method and the one in $[7,16]$ have both met the allowable range of FARs for the 4 sampling times. With the reduction of the values of MDR in $[7,16]$, the values of MDR from our proposed method are growing as time goes on, but the values of MDR obtained in $[7,16]$ are still bigger than the ones obtained by our method. 
Table 1 Comparison of some parameters with different methods

\begin{tabular}{cccccc}
\hline \multicolumn{2}{c}{ Time $(\mathrm{s})$} & 20 & 60 & 100 & 140 \\
\hline & $\mathrm{E}(J(r, k))$ & 0.0766 & 0.1336 & 0.1735 & 0.2052 \\
& $\sigma(J(r, k))$ & 0.0078 & 0.0077 & 0.0076 & 0.0079 \\
Proposed & $J_{t h}(r, k)$ & 0.1078 & 0.1644 & 0.2039 & 0.2368 \\
method & $\eta$ & 4 & 4 & 4 & 4 \\
& $\mathrm{FAR}(\%)$ & 6.25 & 6.25 & 6.25 & 6.25 \\
& $\mathrm{MDR}(\%)$ & 11.10 & 13.73 & 18.57 & 20.30 \\
& $\mathrm{E}(J(r, k))$ & 0.0766 & 0.1336 & 0.1735 & 0.2052 \\
$\mathrm{By}[7,16]$ & $\sigma(J(r, k))$ & 0.0078 & 0.0077 & 0.0076 & 0.0079 \\
& $J_{t h}$ & 0.2449 & 0.2449 & 0.2449 & 0.2449 \\
& $\eta$ & 21 & 14 & 9 & 5 \\
& $\mathrm{FAR}(\%)$ & 0.23 & 0.51 & 1.23 & 4.00 \\
& $\mathrm{MDR}(\%)$ & 65.20 & 51.67 & 37.83 & 25.00 \\
\hline
\end{tabular}

In the simulations, we suppose the fault(s) occurred from $k=60$ to $k=120$. The residual evolution $J(r, k)$ for different failure cases are shown in Figs. 2-4, respectively. Fig. 2 shows the case of the actuator\#1 fault, Fig. 3 shows the case of the sensor\#2 fault, and the case of both the actuator\#1 and the sensor\#1 faults at the same time is shown in Fig. 4. For $J_{t h}(r, k)$ obtained by using (28), and $\mathrm{E}(J(r, k)), \sigma(J(r, k))$ are shown in Table 1. The simulation results show that the residual evolutions are all less than the threshold in the absence of fault.

If actuator $\# 1$ gets faulty from $20 \mathrm{~s}$ to $30 \mathrm{~s}$, then by using different thresholds with our proposed method and the method in referenced literature, the residual evolutions and the residual signal are shown in Fig. 5.

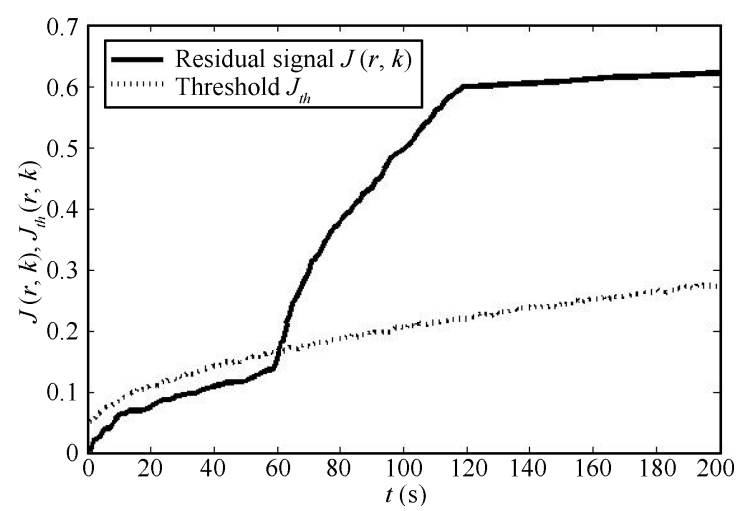

Fig. 2 Evolution of $J(r, k)$ and $J_{t h}(r, k)$ with faulty actuator\#1

From Fig. 4, it can be seen that $J(r, 22)=0.1338>$ $J_{t h}(r, 22)=0.1104$, the fault can be detected in two time steps after its occurrence. The fault can be detected at $22 \mathrm{~s}$ by our proposed method. By $[7,16]$, with the threshold $J_{t h}=0.2449$, from Fig. 5 , it is shown that $J(r, 85)=$ $0.2437<J_{t h}<J(r, 86)=0.2450$. Therefore, the fault can be detected in 66 time steps after its occurrence. It is obvious that our proposed method can detect the fault earlier than the one in the existing literatures as $[7,16]$.

The FAR and MDR of $[7,16]$ shown in Table 1 and Fig. 5 are related with $L_{t h}$. If the fault happens very late within the maximum time step $L_{t h}$, the FAR will be very large, and the MDR will be very large if the fault happens very early when $L_{t h}$ is long. If we set the short $L_{t h}$, the MDR can reduce, but the high FAR cannot be avoided. Colligating the FAR and MDR, our proposed method is superior to $[7,16]$.

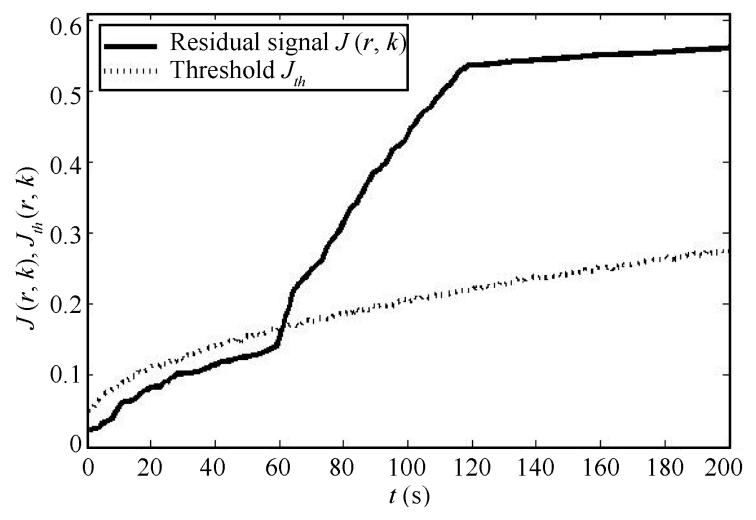

Fig. 3 Evolution of $J(r, k)$ and $J_{t h}(r, k)$ with faulty sensor\#2

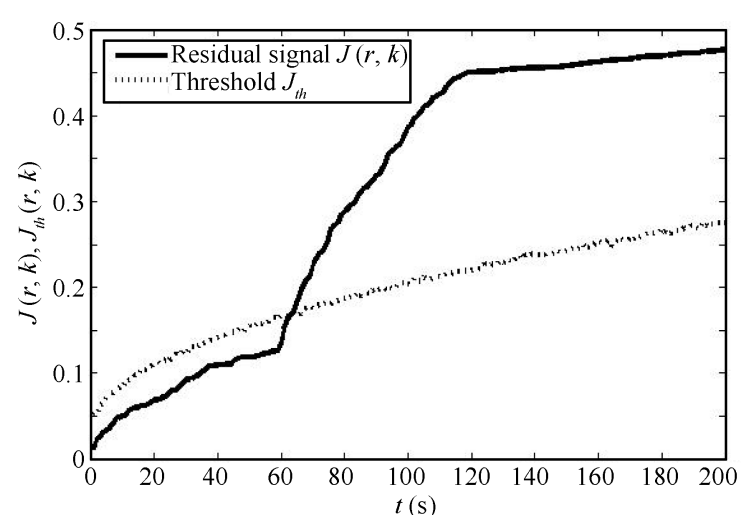

Fig. 4 Evolution of $J(r, k)$ and $J_{t h}(r, k)$ with both actuator\#1 and sensor\#1 faults

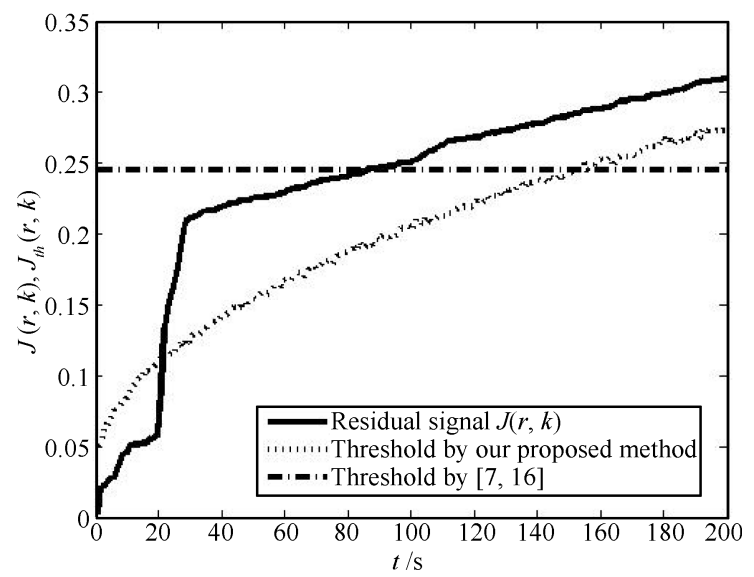

Fig. 5 Evolution of $J(r, k)$ and $J_{t h}(r, k)$ with faulty actuator\#1 obtained by our method and $[7,16]$

Remark 3. A large number of simulation experiments show that the average detection time is $3.7 \mathrm{~s}$ if the fault occurs at $190 \mathrm{~s}$, by using our proposed method. The residual evaluation function and threshold are recalculated after $200 \mathrm{~s}$. So there is no worry over the growth of the MDR value through our proposed method, as the MDR value increases with time. 


\section{Conclusions}

This paper studied the sensors/actuators fault detection for networked control systems with transmission delays. To cope with the time delay, the state observer and predictive control are adopted. After the FD filter was constructed, the optimal $H_{\infty}$ performance index can be achieved. When actuators/sensors failure occurred, one can detect through comparing the residual evaluation function with the threshold. A new threshold computation method that conforms to the actual situation was proposed, then we compare the threshold of FAR/MDR proposed in the paper with the ones in the existing literature method. Finally, numerical simulations were performed and the results verify the effectiveness of the proposed method.

\section{References}

[1] H. S. Park, Y. H. Kim, D. S. Kim, W. H. Kwon. A scheduling method for network-based control systems. IEEE Transactions on Control Systems Technology, vol. 10, no. 3 , pp. 318-330, 2002.

[2] K. C. Lee, S. Lee, M. H. Lee. Remote fuzzy logic control of networked control system via Profibus-DP. IEEE Transactions on Industrial Electronics, vol. 50, no. 4, pp. 784-792, 2003.

[3] J. M. Guerrero, J. Matas, L. G. deVicuna, M. Castilla, J. Miret. Wireless-control strategy for parallel operation of distributed-generation inverters. IEEE Transactions on Industrial Electronics, vol. 53, no. 5, pp. 1461-1470, 2006.

[4] C. B. Jacobina, T. M. Oliveira, E. R. C. da Silva. Control of the single-phase three-leg AC/AC converter. IEEE Transactions on Industrial Electronics, vol. 53, no. 2, pp. 467-476, 2006.

[5] M. Y. Zhong, S. X. Ding, J. Lam, H. B. Wang. An LMI approach to design robust fault detection filter for uncertain LTI systems. Automatica, vol. 39, no. 3, pp. 543-550, 2003.

[6] Y. Q. Wang, H. Ye, S. Ding, G. Z. Wang. Fault detection of networked control systems subject to access constraints and random packet dropout. Acta Automatica Sinica, vol. 35, no. 9, pp. 1230-1234, 2009.

[7] H. Hang, D. X. Xie, D. F. Zhang, Z. Q. Wang. $H_{\infty}$ fault detection for networked control system with Random Markov delays. Information and Control, vol. 39, no. 1, pp. 6-12, 2009.

[8] M. Y. Zhong, Q. L. Han. Fault detection filter design for a class of networked control systems. In Proceedings of the 6th World Congress on Intelligent Control and Automation, IEEE, Dalian, China, pp. 215-219, 2006.

[9] H. J. Fang, H. Ye, M. Y. Zhong. Fault diagnosis of networked control systems. Annual Reviews in Control, vol. 31, no. 1 , pp. 55-68, 2007.

[10] X. Y. Luo, M. J. Shang, C. L. Chen, X. P. Guan. Guaranteed cost active fault-tolerant control of networked control system with packet dropout and transmission delay. International Journal of Automation and Computing, vol. 7, no. 4, pp. 509-515, 2010.

[11] S. B. Li, D. Sauter, C. Aubrun, J. Yamé. Stability guaranteed active fault-tolerant control of networked control systems. Journal of Control Science and Engineering, vol. 2008, Article ID. 189064, 1-9, 2008.

[12] H. Huang, X. D. Han, D. X. Xie, Z. Q. Wang. Active faulttolerant control for networked control systems with packet dropout. Control and Decision, vol. 24, no. 8, pp. 1126-1131, 2009. (in Chinese)
[13] W. Li, J. Q. Zhang, Y. J. Li. Design of robust fault-tolerant controller based on delay model for networked control systems. Control Engineering of China, vol.16, no. 5, pp. 517521, 526, 2009. (in Chinese)

[14] Z. J. Li, L. F. Wang, X. H. Lai, S. H. Xu. Stability of constrained model predictive control for networked control systems with data packet dropout. In Proceedings of IEEE International Conference on Automation and Logistics, IEEE, Jinan, China, pp. 3018-3023, 2007.

[15] Y. Xia, G. P. Liu, M. Fu, D. Rees. Predictive control of networked systems with random delay and data dropout. IET Control Theory and Applications, vol. 3, no. 11, pp. 14761486, 2009.

[16] M. Y. Zhong, H. Ye, P. Shi, G. Wang. Fault detection for Markovian jump systems. IEE Proceedings: Control Theory and Applications, vol. 152, no. 4, pp. 397-402, 2005

[17] J. Liang, L. Y. Qiao, X. Y. Peng. Fault detection based on SIR state estimation and smoothed residual. Acta Electronica Sinica, vol. 35, no. 12A, pp. 32-36, 2007. (in Chinese)

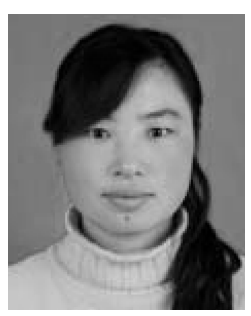

Yu-Yan Zhang received the M. Sc. and $\mathrm{Ph}$. D. degrees from Institute of Electrical Engineering, Yanshan University, China in 2001 and 2008, respectively. She is currently an associate professor in Yanshan University, China.

Her research interests include fault detection and fault-tolerant control, computer measurement and control system.

E-mail: yyzhang@ysu.edu.cn

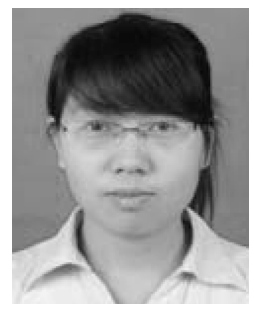

Jun-Ling Zhang received her M.Sc degree in Institute of Electrical Engineering, Yanshan University, China in 2012.

Her research interests include fault detection and fault-tolerant control of networked control systems.

E-mail: Junling111@163.com

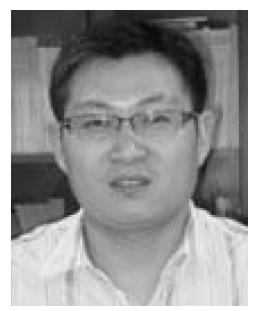

Xiao-Yuan Luo received the M. Sc. and $\mathrm{Ph} . \mathrm{D}$. degrees from Institute of Electrical Engineering, Yanshan University, China in 2001 and 2005, respectively. He is currently a professor in Yanshan University, China.

His research interests include fault detection and fault-tolerant control, and networked control systems.

E-mail: xyluo@ysu.edu.cn (Corresponding author)

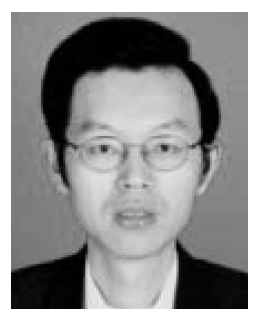

Xin-Ping Guan received his M. Sc. degree in applied mathematics, and his Ph.D. degree in electrical engineering from Harbin Institute of Technology, China in 1991 and 1999 , respectively. He is currently a professor of control theory and control engineering in Yanshan University, China

His research interests include robust congestion control in communication networks, multi-agent systems, and networked control

systems.

E-mail: xpguan@sjtu.edu.cn 